\title{
Focal Adhesion Kinase Is Important for Fluid Shear Stress-Induced Mechanotransduction in Osteoblasts
}

\author{
Suzanne RL Young, ${ }^{1}$ Rita Gerard-O'Riley, ${ }^{1}$ Jae-Beom Kim, ${ }^{2}$ and Fredrick M Pavalko ${ }^{1}$
}

\begin{abstract}
Mechanical loading of bone is important for maintenance of bone mass and structural stability of the skeleton. When bone is mechanically loaded, movement of fluid within the spaces surrounding bone cells generates fluid shear stress (FSS) that stimulates osteoblasts, resulting in enhanced anabolic activity. The mechanisms by which osteoblasts convert the external stimulation of FSS into biochemical changes, a process known as mechanotransduction, remain poorly understood. Focal adhesions are prime candidates for transducing external stimuli. Focal adhesion kinase (FAK), a nonreceptor tyrosine kinase found in focal adhesions, may play a key role in mechanotransduction, although its function has not been directly examined in osteoblasts. We examined the role of FAK in osteoblast mechanotransduction using short interfering RNA (siRNA), overexpression of a dominant negative FAK, and $\mathrm{FAK}^{-1-}$ osteoblasts to disrupt FAK function in calvarial osteoblasts. Osteoblasts were subjected to varying periods oscillatory fluid flow (OFF) from 5 min to $4 \mathrm{~h}$, and several physiologically important readouts of mechanotransduction were analyzed including: extracellular signal-related kinase 1/2 phosphorylation, upregulation of c-fos, cyclooxygenase-2, and osteopontin, and release of prostaglandin $\mathrm{E}_{2}$. Osteoblasts with disrupted FAK signaling exhibited severely impaired mechanical responses in all endpoints examined. These data indicate the importance of FAK for both short and long periods of FSS-induced mechanotransduction in osteoblasts.
\end{abstract}

J Bone Miner Res 2009;24:411-424. Published online on November 3, 2008; doi: 10.1359/JBMR.081102

Key words: integrins, osteopontin, osteoblasts, mechanical loading, stress/strain

\section{INTRODUCTION}

B ONE TISSUE EXISTS in a tightly regulated balance between bone formation and bone resorption, and a key mediator of bone remodeling is the mechanical load that is placed on the tissue. Mechanical loading of bone causes movement of interstitial fluid through the spaces within bone and generates fluid shear stress (FSS) across the surface of the bone cells. Osteoblasts respond to this FSS by controlling expression of proteins involved in bone formation and bone resorption such as cyclooxygenase-2 (COX-2) and prostaglandin E2 ( $\left.\mathrm{PGE}_{2}\right){ }^{(1-4)}$

Focal adhesions, sites of focal contact between the cell and the extracellular matrix, are proposed mechanosensors that connect mechanical signals of FSS from the extracellular matrix with cytoplasmic signaling molecules in a mechanism referred to as mechanotransduction in osteoblasts. ${ }^{(5-9)}$ Focal adhesions are composed of integrins and many other focal adhesion associated proteins such as vinculin, $\alpha$-actinin, and actin filaments. ${ }^{(10-13)}$ Whereas integrins provide a physical connection between the extracellular matrix and the cytoplasm, they lack intrinsic enzymatic activity in their cytoplasmic domains. Therefore, transmission

Dr Kim is an employee of Caliper Life Sciences. All other authors state that they have no conflicts of interest. of the signal is hypothesized to be mediated by nonreceptor tyrosine kinases. Focal adhesion kinase (FAK) is a nonreceptor tyrosine kinase that associates with integrins at focal adhesions. ${ }^{(14)}$ This interaction activates FAK by an autophosphorylation event at tyrosine 397, which provides a binding site for Src and other signaling molecules. ${ }^{(15,16)}$ In addition, the C-terminal domain of FAK can associate with talin and paxillin, which connects the focal adhesion with the actin cytoskeleton. ${ }^{(17,18)}$ Because FAK can associate with several different integrins, it is a key component to the focal adhesion.

Proper function of FAK has been implicated in several cellular processes such as cellular migration, anoikis, and apoptosis. ${ }^{(18-20)}$ Recently the role of FAK in bone differentiation and bone regeneration was examined using a conditional $\mathrm{fak}^{-1-}$ mouse. ${ }^{(21)}$ It was determined that FAK is not required for osteoblast differentiation in vivo, but FAK is needed for appropriate bone regeneration in adult mice. ${ }^{(21)}$ Although these data implicate a role for FAK in bone biology, there is very little known regarding the role of FAK during mechanotransduction. In this study, we investigated the role of FAK as an important mechanosensory component for FSS-induced signaling in osteoblasts. We examined both early and late FSS-induced signaling pathways as measured by the following endpoints: extracellular signal-related kinases (ERK) phosphorylation, COX-2

\footnotetext{
${ }^{1}$ Department of Cellular and Integrative Physiology, Indiana University School of Medicine, Indianapolis, Indiana, USA; ${ }^{2}$ Caliper Life Sciences, Alameda, California, USA.
} 
upregulation, $\mathrm{PGE}_{2}$ release, c-fos upregulation, and osteopontin (OPN) upregulation. Furthermore, we used osteoblasts from multiple sources and three different methods to disrupt FAK activity to test our hypothesis. Using siRNA technology to target FAK expression, we found that reduced FAK expression resulted in a decrease in both the early and late FSS-induced signaling pathways in osteoblasts. In addition, a dominant negative FAK protein FAK-related nonkinase (FRNK), is endogenously expressed in osteoblasts. Overexpression of FRNK disrupted FAK activation, which also resulted in a reduced response to FSS in osteoblasts. Finally, $\mathrm{FAK}^{-1-}$ osteoblasts were also examined and exhibited a diminished response to FSS. Importantly, re-expression of FAK in the $\mathrm{FAK}^{-/-}$osteoblasts rescued FSS-induced mechanotransduction as measured by COX-2 upregulation and OPN expression.

\section{MATERIALS AND METHODS}

\section{Cell culture}

Osteoblast-like cells were isolated from rat calvaria as described previously ${ }^{(22)}$ and are referred to as osteoblasts throughout the text. Briefly, calvaria from 10-13 neonatal rats (birth to day 3 ) were isolated aseptically, minced, and digested consecutively for 5,20 , and $45 \mathrm{~min}$ at $37^{\circ} \mathrm{C}$ in $0.2 \%$ collagenase $\mathrm{P}-0.25 \%$ trypsin. Cells released in the second and third digests were pooled and cultured in MEM supplemented with $10 \%$ FCS and antibiotics. Experiments were performed with passages 2-5.

Immortalized $\mathrm{FAK}^{-/-}$(clones 4F1, 1D8) and $\mathrm{FAK}^{+/+}$ (clones 1C7, 1E11) osteoblast clones were established as described in Kim et al. ${ }^{(21)}$ Briefly, primary cells from mouse calvaria were harvested from fak floxed/floxed $; p 53^{-/-}$conditional knockout mice and infected using CMV-Cre adeno-

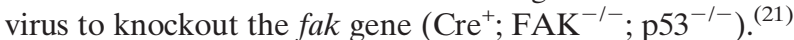
Single cell cloning was performed using a limiting dilution, and individual clones were established as cell lines. Control calvarial osteoblasts harvested from fak floxedfloxed mice that were not exposed to Cre recombinase $\left(\mathrm{Cre}^{-}\right.$; $\mathrm{FAK}^{\text {floxed/floxed; }}$ p53 ${ }^{-1-}$ ) were established in a similar fashion. ${ }^{(21)}$ The mouse clones were cultured in MEM supplemented with $10 \%$ FCS and antibiotics. Both $\mathrm{FAK}^{+/+}$ and $\mathrm{FAK}^{-1-}$ clones express the osteoblast markers OPN and alkaline phosphatase (ALP) and contribute to fetal skeletogenesis in vivo. ${ }^{(21)}$ Experiments were performed with passages 2-7 to maintain continuity between experiments (passage 0 is defined as immediately after clonal selection).

\section{Constructs}

Full-length green fluorescent protein (GFP)-tagged FAK (FAK-GFP) and GFP tagged FRNK (FRNK-GFP) were gifts of Dr Lewis Romer (Johns Hopkins University). Briefly, the GFP-FAK cDNA and GFP-FRNK cDNA were cloned into EcoRV and BamHI sites of a pVQcmv $\mathrm{K}-\mathrm{NpA}$ vector. Viral preparations were commercially manufactured and purified to $4 \times 10^{10}$ plaque-forming units/ml (ViraQuest, North Liberty, IA, USA). GFP-only viral particles were prepared as described previously. ${ }^{(23)}$

\section{Adenoviral infection}

Osteoblasts were seeded at $1.2-1.5 \times 10^{5}$ cells per slide and were infected with FRNK-GFP, FAK-GFP, or GFP only MOI 500 in 10\% MEM for $4 \mathrm{~h}$ (see Constructs). Complete media were added, and cells were incubated overnight at $37^{\circ} \mathrm{C}$ with $5 \% \mathrm{CO}_{2}$. The next day, media were removed and replaced with $0.1 \%$ MEM for an overnight incubation at $37^{\circ} \mathrm{C}$ with $5 \% \mathrm{CO}_{2}$. On the third day, infected cells were exposed to oscillatory fluid flow.

\section{siRNA transfection}

SMART pool siRNA oligonucleotides targeting rat FAK mRNA (GenBank accession number NM_013018) were obtained from Dharmacon (Lafayette, CO, USA). For siRNA transfections, osteoblasts were plated at $30 \%$ confluency in a 6-well dish. The following day, cells were transfected with siRNA using Lipofectamine 2000 (Invitrogen) according to manufacturer's protocol. Two control transfections were performed: one with Lipofectamine 2000 alone and the other with a non targeting scramble siRNA control (Dharmacon). Cells were used for experiments 4-7 days after transfection.

\section{Oscillatory fluid flow conditions}

Cells were subjected to oscillatory fluid shear stress (1012 dynes $/ \mathrm{cm}^{2}$ ) in parallel plate flow chambers at $37^{\circ} \mathrm{C}$ using a previously described fluid flow device. ${ }^{(24)}$ Hard-walled tubing was used to connect the pump to the chamber inlet, and a reservoir was attached to the outlet to allow for movement of the fluid and exchange of $5 \% \mathrm{CO}_{2}$. This system subjects cells to oscillating fluid flow at a frequency of $0.5 \mathrm{~Hz}$. Static controls were held in cell culture dishes at $37^{\circ} \mathrm{C}$ with $5 \% \mathrm{CO}_{2}$.

\section{Immunoblot analysis}

Cells subjected to static and oscillatory fluid flow were harvested in SDS sample buffer, and protein concentrations were determined using the amido black method. ${ }^{(25)}$ Equal amounts of protein $(20 \mu \mathrm{g})$ were loaded onto SDSPAGE gels for separation and transferred to PVDF (nitrocellulose was used when probing for phospho-Pyk2). The following primary and secondary antibodies were used: ERK1/2 (Santa Cruz), phospho-ERK1/2 (Santa Cruz), COX-2 (Santa Cruz), OPN (Santa Cruz), c-fos (Santa Cruz), FAK (Upstate), phospho-FAK Y397 (BD,), Pyk2 (BD), phospho-Pyk2 Y402 (BD,), vinculin (Sigma), horseradish peroxidase (HRP)-conjugated donkey antirabbit and HRP-conjugated donkey anti-goat (Jackson Immunoresearch, West Grove, PA, USA), and HRP-conjugated donkey anti-goat (Santa Cruz). Densitometry was quantitated using Image $\mathbf{J}$ software $(\mathrm{NIH})$.

\section{Statistical analysis}

Statistical significance was assessed by one-tailed $t$ tests with a $p$ value of $<0.05$ interpreted as statistically significant. 


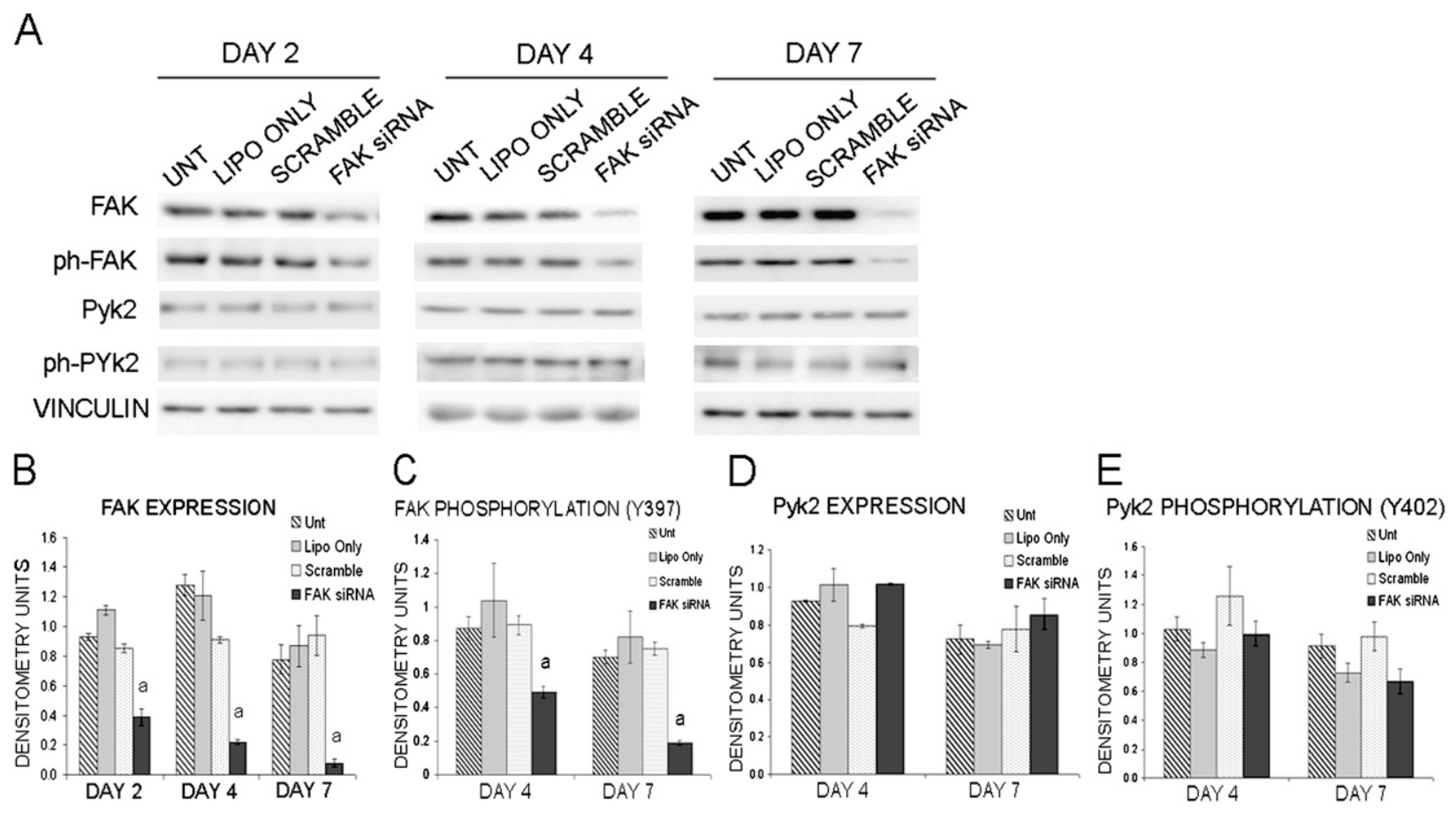

FIG. 1. Transfection of FAK siRNA in osteoblasts. (A) Western blot analysis of FAK and Pyk2 expression and activation (phosphorylation of Y397, Y402) in osteoblasts treated with lipofectamine only (Lipo Only), $50 \mathrm{nM}$ scrambled control siRNA (Scramble), or 50 nM FAK siRNA smart pool (FAK siRNA) compared with untreated osteoblasts (Unt). Vinculin was analyzed as a loading control. (B and C) Quantification of FAK expression and FAK activation as measured by phosphorylation of Y397 expressed as raw densitometry units ( ${ }^{\mathrm{a}} p<0.05$ ). (D and E) Quantification of Pyk2 expression and Pyk2 activation as measured by phosphorylation of Y402 expressed as raw densitometry units. Error bars represent SE.

\section{RESULTS}

\section{Inhibition of FAK activity in osteoblasts}

Three complimentary approaches were used to evaluate the role of FAK in mechanotransduction in osteoblasts. First, to evaluate the role of FAK in osteoblast mechanotransduction, primary osteoblasts isolated from rat calvaria were treated with FAK siRNA $(25-75 \mathrm{nM})$ to downregulate FAK expression. FAK expression was reduced by $54 \%, 76 \%$, and $90 \%$ after treatment with FAK siRNA at $50 \mathrm{nM}$ on days 2, 4, and 7 after transfection, respectively (Figs. $1 \mathrm{~A}$ and $1 \mathrm{~B}$ ). In contrast, control osteoblasts transfected with $50 \mathrm{nM}$ of a nontargeting scrambled siRNA or osteoblasts exposed to transfection reagent showed no reduction in FAK expression compared with untreated osteoblasts (Figs. 1A and 1B). Targeting of FAK using siRNA resulted in a $45 \%$ and $75 \%$ reduction of activated FAK as measured by phosphorylation of FAK at tyrosine 397 (Y397) on days 4 and 7 compared with the scramble control, which did not affect FAK activation (Figs. 1A and 1C). Therefore, subsequent experiments were carried out at 4-7 days after transfection. In addition, we examined the affect of FAK siRNA on Pyk2 expression and activation. We determined that decreased expression/activation of FAK through FAK siRNA did not result in an increase in Pyk2 expression or activation as measured by phosphorylation of tyrosine 402 (Y402) (Figs. 1D and 1E).
As a second, alternative method to disrupt FAK signaling in osteoblasts, the dominant negative protein FRNK was overexpressed in primary osteoblasts. FRNK is an autonomously expressed protein that is a negative regulator of FAK activity and is endogenously expressed in primary rat osteoblasts. ${ }^{(26,27)} \mathrm{We}$ determined that infection with FRNK-GFP resulted in an $\sim 3$.2-fold increase in expression of total FRNK in osteoblasts $48 \mathrm{~h}$ after infection (Figs. 2A and 2B). Importantly, FAK activation as measured by Y397 phosphorylation was decreased by 80\% in these cells, indicating that overexpression of FRNK was effective at inhibiting activation of FAK in osteoblasts (Fig. 2B). Again we examined the expression and activation of Pyk2 in response to decreased FAK activation. It was determined that Pyk2 expression and activation did not change in osteoblasts overexpressing FRNK (Fig. 2C).

A third approach to assess the role of FAK in mechanotransduction used immortalized $\mathrm{FAK}^{-/-}$osteoblasts harvested from fak floxed/floxed conditional knockout mice exposed to Cre recombinase ${ }^{(21)}$ (Fig. 2D). Two separate $\mathrm{FAK}^{-1-}$ clones $(4 \mathrm{~F} 1,1 \mathrm{D} 8)$ were analyzed as well as two control clones $(1 \mathrm{C} 7,1 \mathrm{E} 11)$, which were harvested from fak $^{\text {floxedfloxed }}$ mice that were not exposed to Cre recombinase. ${ }^{(21)}$ The FAK $^{-1-}$ clones do not express FAK as determined by Western blot analysis (Fig. 3A). In addition, we examined the expression of protein tyrosine kinase 2 (Pyk2), which is a member of the FAK family of protein kinases. ${ }^{(18,28)}$ We determined that the $\mathrm{FAK}^{-1-}$ clones did 

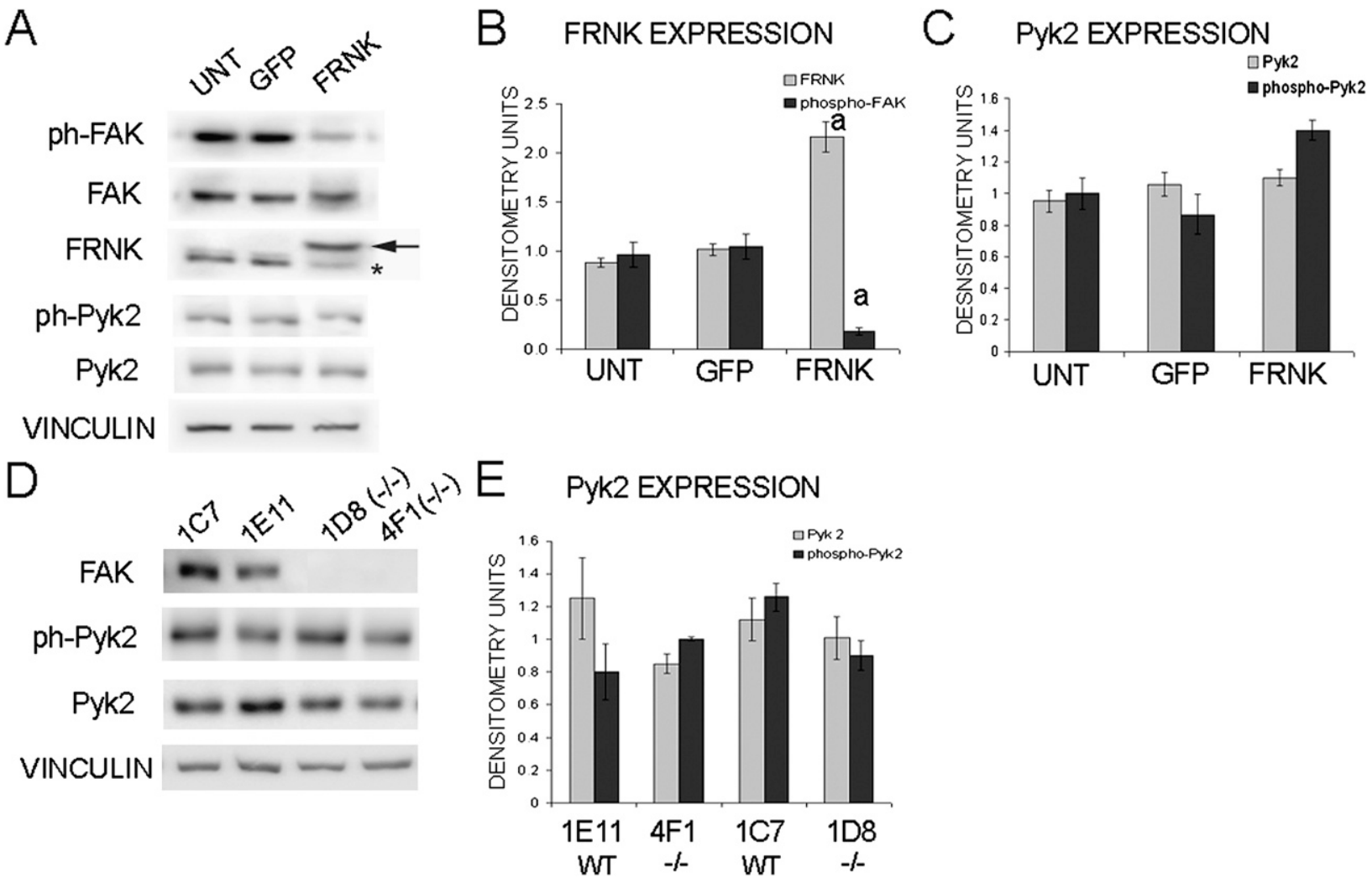

FIG. 2. Analysis of osteoblasts infected with FAK-related nonkinase (FRNK) and FAK ${ }^{+/+}$and FAK ${ }^{-/-}$osteoblasts. (A) Western blot analysis of FAK activation (phosphorylation at Y397), FRNK expression, Pyk2 expression, and activation (phosphorylation at Y402) in osteoblasts infected with GFP only control (GFP) or FRNK-GFP (FRNK) compared with uninfected osteoblasts. Arrow indicates GFPFRNK; asterisk indicates endogenous FRNK. Vinculin was analyzed as a loading control. (B) Quantification of FRNK expression and FAK activation after infection as compared with uninfected osteoblasts expressed in raw densitometry units. (C) Quantification of Pyk2 expression and activation after infection compared with uninfected osteoblasts expressed in raw densitometry units. (D) Western blot analysis of control osteoblasts clone (1C, 1E11) and $\mathrm{FAK}^{-1-}$ osteoblast clones (1D8, 4F1) shows no FAK expression wildtype levels of Pyk2 expression in the $\mathrm{FAK}^{-1-}$ clones. $\mathrm{FAK}^{-l-}$ clones exhibit similar levels of activated Pyk2 as measured by phosphorylation at Y402

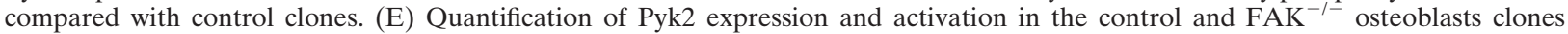
expressed in raw densitometry units $\left({ }^{\mathrm{a}} p<0.05\right)$. Error bars represent SE.

not exhibit a significant difference in Pyk2 expression as analyzed by Western blot (Figs. 2D and 2E). Furthermore, we examined activation of Pyk2 as measured by phosphorylation of Pyk2 at Y402. We determined that there is not a significant difference in Pyk2 activation in the $\mathrm{FAK}^{-1-}$ clones we examined (Figs. 2D and 2E).

\section{Reduced shear-induced ERK phosphorylation in FAK-deficient osteoblasts}

To determine the role of FAK in osteoblast mechanotransduction after short periods of FSS, we first analyzed the impact of FSS on activation of ERK signaling. ERK becomes phosphorylated in osteoblasts within 5 min of oscillatory fluid flow (OFF) and begins to decline after 30 min of OFF. ${ }^{(8,29-35)}$ To determine whether FAK is needed for OFF-stimulated ERK phosphorylation, we exposed osteoblasts to 5, 15, and $30 \mathrm{~min}$ of OFF in FAK siRNAtransfected osteoblasts. Protein was harvested, and Western blot analysis was used to measure the relative amount of phosphorylated ERK in the samples. In FAK siRNA- treated osteoblasts, ERK phosphorylation was increased only 1.3 -fold after 5 min of OFF compared with nonflowed cells. In contrast, osteoblasts treated with the control scrambled siRNA exhibited a 3.6-fold increase in ERK phosphorylation after $5 \mathrm{~min}$ of OFF (Fig. 3A). Other controls including lipofectamine only and untreated osteoblasts showed increases of 3.7- and 3.0-fold in ERK phosphorylation at $5 \mathrm{~min}$, respectively (Fig. 3A). Thus, the FAK siRNA-treated osteoblasts showed a $\sim 64 \%$ decrease in ERK phosphorylation after 5 min of OFF. After $15 \mathrm{~min}$ of OFF, the FAK siRNA-treated osteoblasts had a 1.5-fold increase in ERK phosphorylation, whereas the scramble control osteoblasts exhibited a 4.3-fold increase, which corresponds to a $65 \%$ reduction in ERK phosphorylation. After $30 \mathrm{~min}$ of OFF, ERK phosphorylation in FAK siRNA-treated osteoblasts was not significantly different from the control (scrambled siRNA) osteoblasts.

We also examined ERK phosphorylation in osteoblasts overexpressing FRNK and found that uninfected osteoblasts and GFP-infected osteoblasts both showed a 2.9-fold increase in ERK phosphorylation after $5 \mathrm{~min}$ of OFF 
A

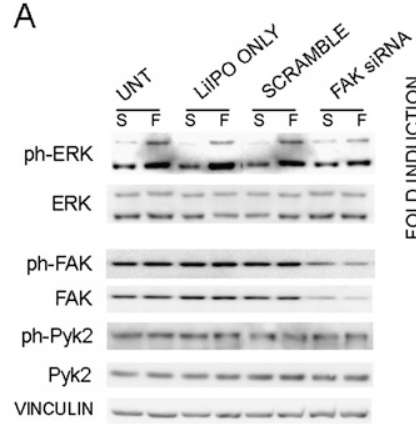

B

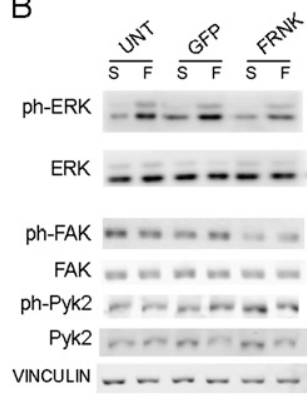

C

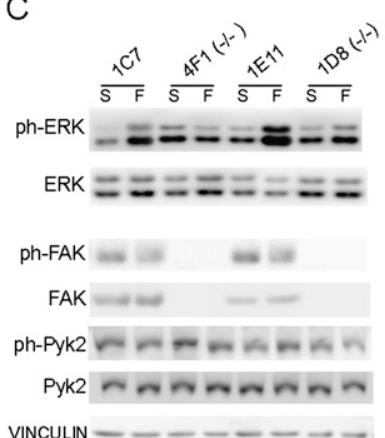

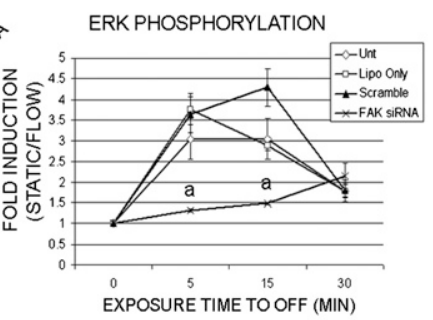

ERK PHOSPHORYLATION

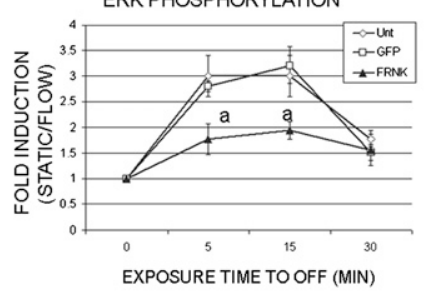

EXPOSURE TIME TO OFF (MIN)

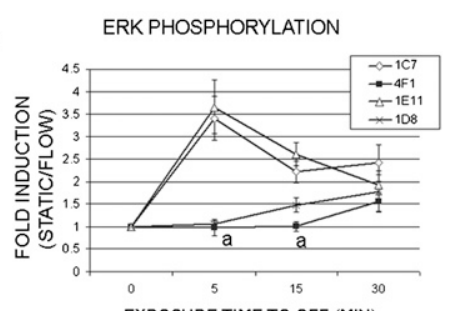

EXPOSURE TIME TO OFF (MIN)
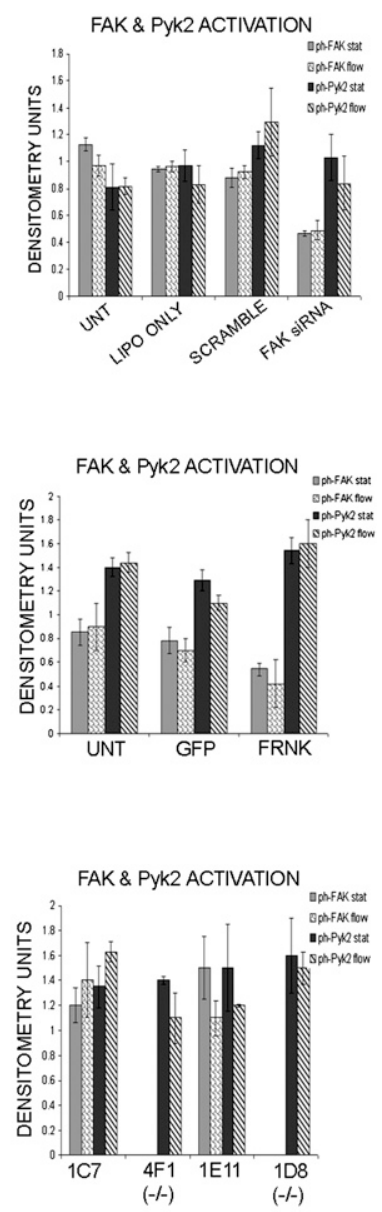

FIG. 3. Reduced ERK activation in osteoblasts exhibiting disrupted FAK activation. Western blot analysis of ERK activation, FAK and Pyk2 expression, and activation after $5 \mathrm{~min}$ of OFF or $5 \mathrm{~min}$ of static culture conditions (S, static; F, flow). Vinculin was analyzed as a loading control. Line graphs represent ERK activation as measured in fold induction (flow/static) after 5, 15, and 30 min of OFF. Quantification of FAK and Pyk2 activation shown in bar graphs. (A) Osteoblasts treated with FAK siRNA exhibited decreased ERK activation after exposure to 5,15 , and $30 \mathrm{~min}$ of OFF. (B) Osteoblasts overexpressing FRNK also exhibited decreased ERK activation after 5, 15, and $30 \mathrm{~min}$ of OFF. (C) $\mathrm{FAK}^{-1-}$ osteoblasts exhibited reduced ERK activation after 5, 15, and $30 \mathrm{~min}$ of OFF. Error bars represent $\mathrm{SE}\left({ }^{\mathrm{a}} p<0.05\right)$.
(Fig. 3B). The FRNK overexpressing osteoblasts showed only a 1.6-fold increase in ERK phosphorylation after $5 \mathrm{~min}$ of OFF (Fig. 3B). After 15 min of OFF, the control osteoblasts exhibited a 3.2- to 4.0-fold increase in ERK phosphorylation, whereas osteoblasts overexpressing FRNK exhibited a 1.9-fold increase (Fig. 3B). Similar to the FAK siRNA-treated osteoblasts, the FRNK overexpressing osteoblasts did not show a significant difference in ERK phosphorylation after $30 \mathrm{~min}$ of OFF compared with the uninfected and GFP-infected controls (Fig. 3B).

ERK phosphorylation was also examined in the $\mathrm{FAK}^{-/-}$ osteoblast clones. After 5 min of OFF, $\mathrm{FAK}^{-1-}$ clone $4 \mathrm{~F} 1$ exhibited no increase in ERK phosphorylation, whereas $\mathrm{FAK}^{-1-}$ clone $1 \mathrm{D} 8$ showed a small but statistically significant 1.1-fold increase (Fig. 3C). Both control clones, 1C7 and 1E11, exhibited at least a 3.4-fold increase in ERK phosphorylation at $5 \mathrm{~min}$ (Fig. 3C). After $15 \mathrm{~min}$ of OFF, the two $\mathrm{FAK}^{-1-}$ clones showed a 1.0- to 1.6-fold increase in ERK phosphorylation, whereas the control clones exhibited a 2.2- to 2.6-fold increase, respectively (Fig. 3C). Similar to the FAK siRNA-treated osteoblasts, both $\mathrm{FAK}^{-1-}$ osteoblast clones showed no difference in ERK activation after $30 \mathrm{~min}$ of OFF compared with control
FAK-expressing clones (Fig. 3C). Thus, the FAK ${ }^{-1-}$ clones exhibited a $43-77 \%$ reduction in ERK phosphorylation after 5 and 15 min of OFF (Fig. 3C). These data suggest that FAK is important for FSS-stimulated ERK phosphorylation in osteoblasts.

In addition to ERK activation, we examined the expression and activation of both FAK and Pyk2 in response to these short periods of OFF in primary rat osteoblasts and the immortalized mouse osteoblast clones. We determined that FAK and Pyk2 expression was not significantly changed in response to 5,15 , or $30 \mathrm{~min}$ of $\mathrm{OFF}$ as determined by Western blot analysis (data not shown). Furthermore, FAK and Pyk2 activation was not significantly changed in response to 5, 15, or $30 \mathrm{~min}$ of OFF (Fig. 3, graphs).

\section{FAK is needed for appropriate OFF stimulated $c$-fos upregulation in osteoblasts}

In osteoblasts, c-fos is upregulated on stimulation of short periods of FSS and may play a role in bone remodeling by transcriptional activation of matrix proteins. ${ }^{(7,36-41)}$ To study the role FAK plays in c-fos regulation by FSS, we exposed osteoblasts to $30 \mathrm{~min}$ of OFF or maintained the cells in static 


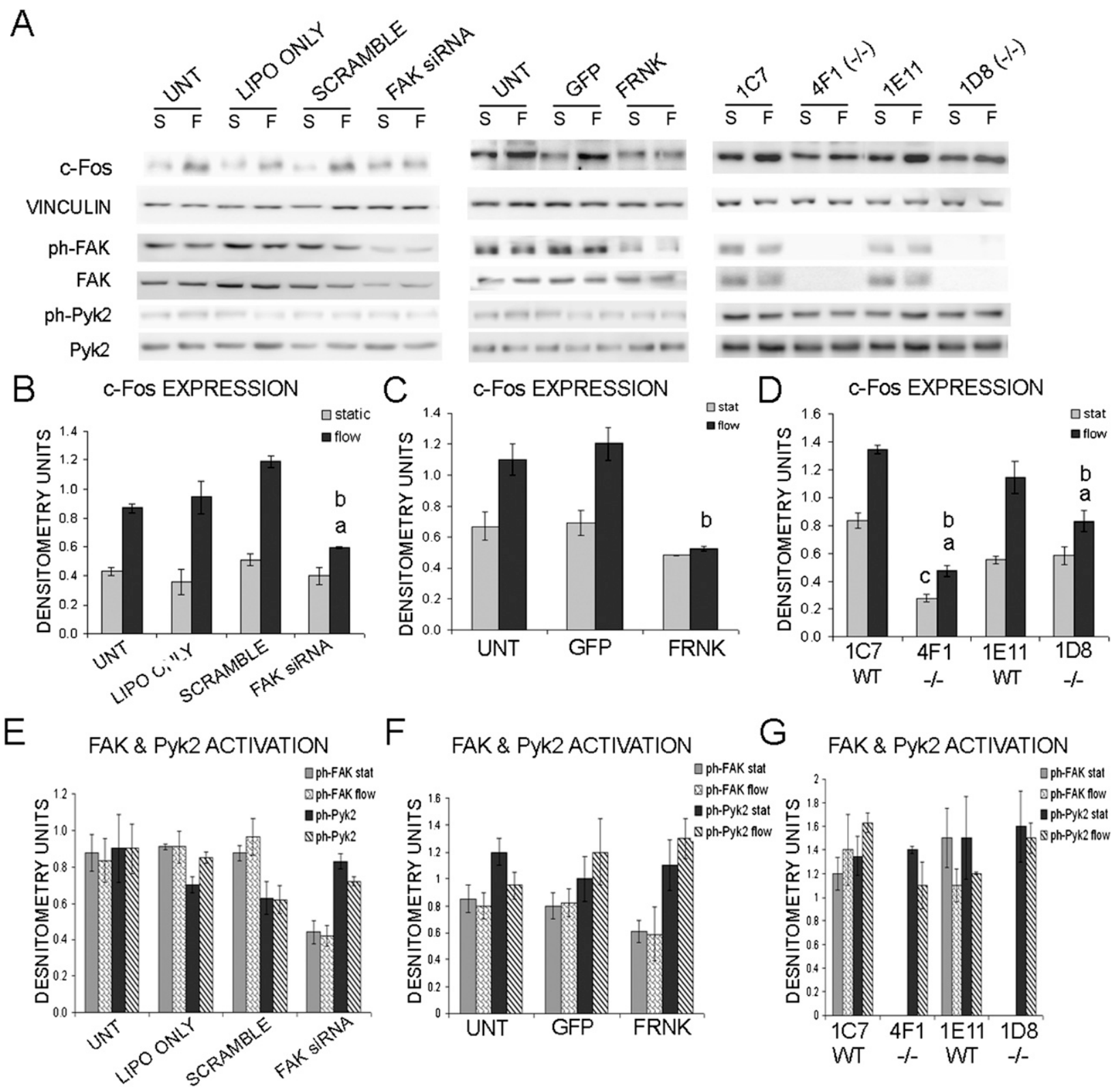

FIG. 4. Reduced induction of c-fos by OFF in osteoblasts exhibiting decreased FAK activation. (A) Western blot analysis of c-fos expression, FAK and Pyk2 expression, and activation after $30 \mathrm{~min}$ of static culture condition or 30 min of OFF ( $\mathrm{S}$, static; F, flow) in FAK siRNA-treated osteoblasts, FRNK overexpressing osteoblasts, and FAK ${ }^{-1-}$ osteoblasts. Vinculin was analyzed as a loading control. (B-D) Graph represents quantification of c-fos expression expressed in raw densitometry units. (E-G) Graph represents quantification of FAK and Pyk2 activation expressed in raw densitometry units. ${ }^{a}$ Statistically significant difference between static and flow. ${ }^{b}$ Statistically significant difference among the flowed samples. ${ }^{\mathrm{c}}$ Statistically significant difference among the static samples $\left({ }^{\mathrm{a}, \mathrm{b}, \mathrm{c}} p<0.05\right)$. Error bars represent SE.

culture conditions. Protein was harvested, and Western blot analysis was used to measure the relative c-fos expression. In lipofectamine only, scramble siRNA, and untransfected osteoblasts, we observed a 2.0- to 2.6-fold increase in c-fos expression on $30 \mathrm{~min}$ of OFF (Fig. 4A). However, in FAK siRNA-treated osteoblasts, c-fos expression was significantly reduced (1.4-fold increase) after $30 \mathrm{~min}$ of OFF (Fig. 4A). Whereas this is a significant increase in c-fos expression compared with FAK siRNA-treated osteoblasts maintained in static culture conditions, the level of upregulation was decreased by $50 \%$ compared with scramble siRNA osteoblasts exposed to OFF.

In FRNK overexpressing osteoblasts, we observed an insignificant increase in c-fos expression on $30 \mathrm{~min}$ of $\mathrm{OFF}$ compared with static culture conditions (Fig. 4B). In contrast we observed a 1.8- and 1.7-fold increase in c-fos expression in the uninfected and GFP-infected control osteoblasts, respectively (Fig. 4B). The level of c-fos expression in the FRNK overexpressing cells was decreased 51-55\% compared with the control osteoblasts exposed to $30 \mathrm{~min}$ of OFF (Fig. 4B). 
A
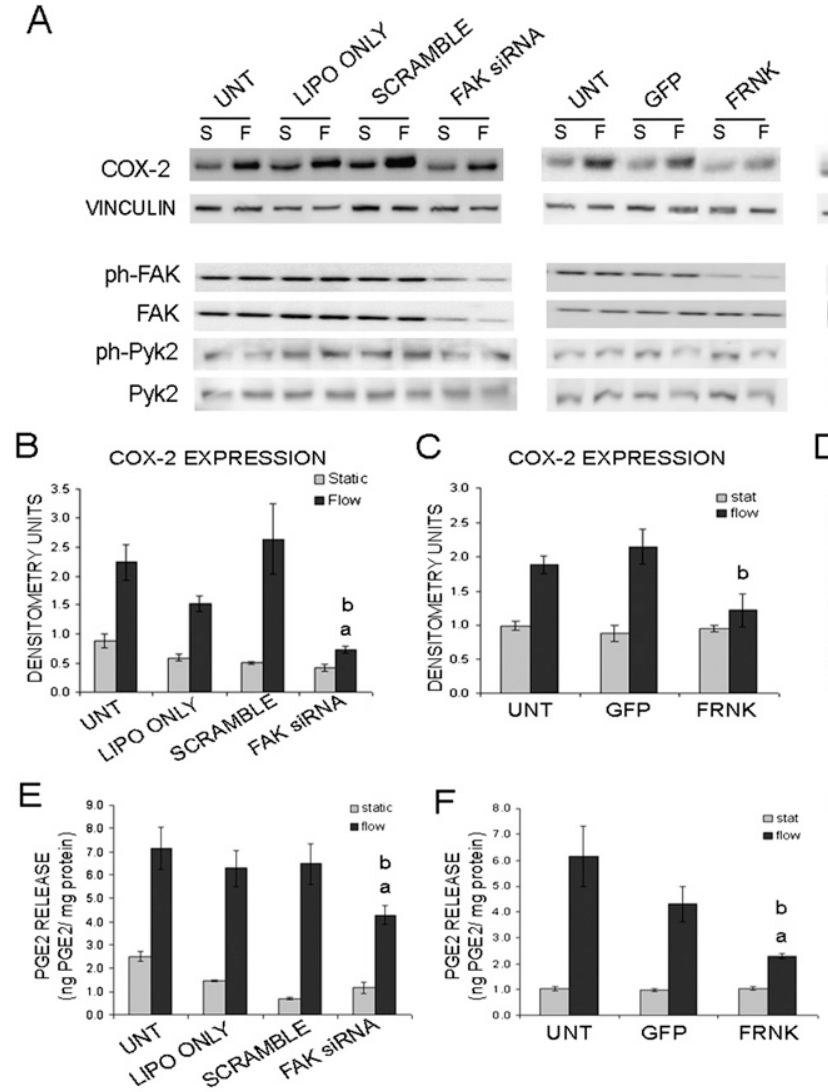

$\mathrm{H}$
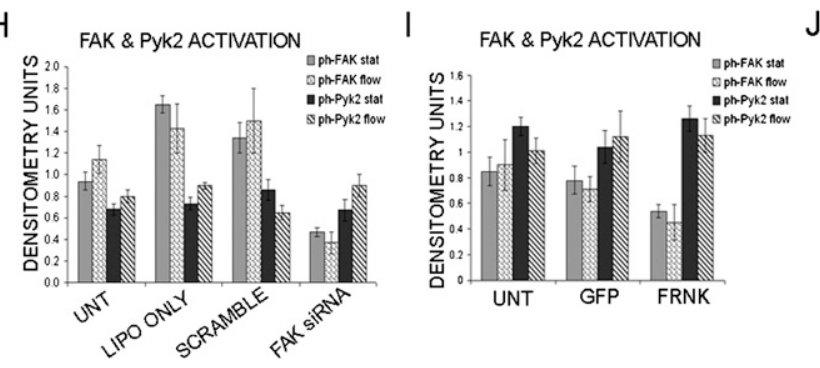
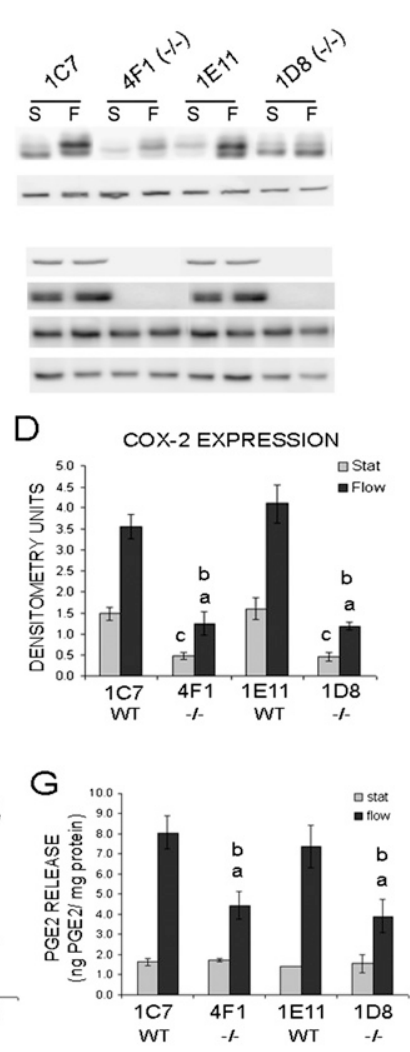

$J$

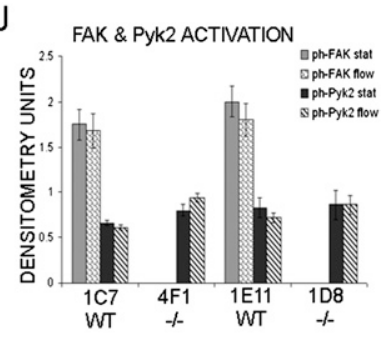

FIG. 5. Decreased COX-2 induction and $\mathrm{PGE}_{2}$ release in response to $\mathrm{OFF}$ in osteoblasts exhibiting disrupted FAK activation. (A) Western blot analysis of COX-2 expression, FAK and Pyk2 expression, and activation after $4 \mathrm{~h}$ of static culture conditions or $4 \mathrm{~h}$ of OFF (S, static; F, flow). Vinculin was analyzed as a loading control. (B-D) Quantification of COX2 expression expressed in raw densitometry units. (E-G) Quantification of $\mathrm{PGE}_{2}$ release as measured by ELISA in osteoblasts exposed to $4 \mathrm{~h}$ of static culture conditions or $4 \mathrm{~h}$ of OFF. (H-J) Quantification of FAK and Pyk2 activation expressed in raw densitometry units. ${ }^{\text {a }}$ Statistically significant difference between static and flow. 'btatistically significant difference among the flowed samples. ${ }^{\text {CStatistically significant }}$ difference among the static samples $\left({ }^{\mathrm{a}, \mathrm{b}, \mathrm{c}} p<0.05\right)$. Error bars represent SE.
The $\mathrm{FAK}^{-1-}$ clones exhibited a 1.4- to 1.6-fold increase in c-fos expression on $30 \mathrm{~min}$ of OFF (Fig. 4C), whereas the control osteoblast clones exhibited a 1.7- to 2.1-fold increase in c-fos expression (Fig. 4C). Although the FAK ${ }^{-1-}$ clones showed a significant increase in c-fos expression after $30 \mathrm{~min}$ of OFF compared with static culture conditions, the level of expression of c-fos after exposure to shear stress was decreased by $50-65 \%$ compared with control clones (Fig. 4C). This data further implicates the importance of FAK for FSS stimulated mechanotransduction in osteoblasts.

We examined the expression and activation of both FAK and Pyk2 in response to $30 \mathrm{~min}$ of OFF in primary rat osteoblasts and the immortalized mouse osteoblast clones. We determined that FAK and Pyk2 expression was not significantly changed in response to $30 \mathrm{~min}$ of OFF as determined by Western blot analysis (data not shown). Furthermore, FAK and Pyk2 activation was not significantly changed in response to $30 \mathrm{~min}$ of OFF (Figs. 4E-4G).

\section{Diminished $C O X-2$ induction and $P G E_{2}$ release on $4 \mathrm{~h}$ of OFF in FAK-deficient osteoblasts}

Osteoblasts increase COX-2 expression and increase the release of $\mathrm{PGE}_{2}$ in response to exposure to longer periods of FSS (2-4 h), which stimulates bone formation by promoting osteoblast differentiation. ${ }^{(7,42-46)}$ We examined the ability of FAK-deficient osteoblasts to regulate COX-2 expression and $\mathrm{PGE}_{2}$ release on exposure to FSS by exposing the osteoblasts to $4 \mathrm{~h}$ of OFF or maintaining the cells in static culture conditions. Media was collected to measure $\mathrm{PGE}_{2}$ secretion, and protein was harvested for Western blot analysis of COX-2. We found that the untransfected, lipofectamine only, and scramble control treated cells exhibited at least a 2.5-fold increase in COX-2 expression (Figs. 5A and 5B). In contrast, the FAK siRNA-treated osteoblasts exhibited a significantly lower increase in COX-2 expression after $4 \mathrm{~h}$ of OFF (Figs. 5A and 5B). Thus, we saw a $37-62 \%$ decrease in COX-2 upregulation on $4 \mathrm{~h}$ of OFF in 
FAK siRNA-treated osteoblasts compared with controls. In addition, the level of COX-2 expression was decreased 52$72 \%$ in FAK siRNA-treated osteoblasts compared with the control osteoblasts exposed to $4 \mathrm{~h}$ of OFF. The reduced COX-2 upregulation in FAK siRNA-treated osteoblasts translated into a decrease in the amount of $\mathrm{PGE}_{2}$ released as measured by ELISA (Fig. 5F). Untransfected, lipofectamine only, and the scramble control treated osteoblasts released 6.5-7.1 ng/mg of $\mathrm{PGE}_{2}$, whereas the FAK siRNA-treated osteoblasts released $\sim 4.3 \mathrm{ng} / \mathrm{mg}$ after $4 \mathrm{~h}$ of OFF (Fig. $5 \mathrm{~F}$ ). This represents a statistically significant $34 \%$ reduction in $\mathrm{PGE}_{2}$ release compared with the scramble control treated osteoblasts exposed to $4 \mathrm{~h}$ of OFF (Fig. 5F).

In FRNK overexpressing osteoblasts, COX-2 upregulation was also reduced compared with GFP-infected and uninfected osteoblasts. The uninfected and GFP-infected osteoblasts exhibited a 1.9- and 2.4-fold increase in COX-2 expression after $4 \mathrm{~h}$ of OFF, whereas the FRNK overexpressing osteoblasts exhibited only a 1.2-fold increase in COX-2 expression after $4 \mathrm{~h}$ of OFF, which was not a significant increase in COX-2 expression (Figs. 5A and 5C). In addition to COX-2 upregulation, we also examined $\mathrm{PGE}_{2}$ release in the FRNK overexpressing cells. Uninfected and GFP-infected osteoblasts released $\sim 4.3-6.2 \mathrm{ng} / \mathrm{mg}$ of $\mathrm{PGE}_{2}$, whereas the FRNK overexpressing osteoblasts released $\sim 2.3 \mathrm{ng} / \mathrm{mg}$ of $\mathrm{PGE}_{2}$ after $4 \mathrm{~h}$ of OFF (Fig. 5G). This corresponds to a $\sim 47 \%$ decrease in $\mathrm{PGE}_{2}$ secretion on stimulation by $4 \mathrm{~h}$ of OFF in osteoblasts, which was statistically significant (Fig. 5G).

In the $\mathrm{FAK}^{-1-}$ osteoblasts, we observed a significant increase in COX-2 expression on exposure to $4 \mathrm{~h}$ of OFF (Figs. $5 \mathrm{~A}$ and 5D). However, the expression level of COX-2 in both $\mathrm{FAK}^{-1-}$ clones was $\sim 65 \%$ lower than the expression of COX-2 in the control clones in both static samples and samples exposed to OFF (Figs. 5A and 5D). Therefore, whereas we did see an increase in COX-2 expression in the $\mathrm{FAK}^{-1-}$ osteoblasts, this increase was significantly less than what we observed in the control osteoblasts. These data indicate that FAK is needed for appropriate induction of COX-2 in osteoblasts, and impaired FAK signaling results in diminished upregulation of COX-2 in response to OFF.

$\mathrm{PGE}_{2}$ release was also examined in the control and $\mathrm{FAK}^{-1-}$ clones. The control clones released $\sim 7.4-8.1 \mathrm{ng} /$ mg of $\mathrm{PGE}_{2}$ after $4 \mathrm{~h}$ of OFF, whereas the $\mathrm{FAK}^{-1-}$ released 3.9-4.4 ng/mg of $\mathrm{PGE}_{2}$ (Fig. 5H). The $\mathrm{FAK}^{-1-}$ clones exhibited a statistically significant decrease of $\sim 46 \%$ in $\mathrm{PGE}_{2}$ secretion after $4 \mathrm{~h}$ of OFF (Fig. $5 \mathrm{H}$ ).

Changes in FAK and Pyk2 expression were examined after $4 \mathrm{~h}$ of OFF in both the primary rat osteoblasts and the immortalized mouse osteoblast clones. We did not observe a significant change in FAK or Pyk2 expression on exposure to $4 \mathrm{~h}$ of OFF as determined by Western blot analysis (data not shown). In addition, we did not observe a significant change in FAK or Pyk2 activation after $4 \mathrm{~h}$ of OFF (Figs. 5H-5J).

\section{FSS-induced upregulation of OPN is reduced in FAK-deficient osteoblasts}

OPN is a protein involved in bone remodeling, and it is upregulated in osteoblasts exposed to FSS followed by an overnight incubation. ${ }^{(34,39,47)}$ We examined the ability of osteoblasts to upregulate OPN in FAK-deficient cells. Osteoblasts were exposed to $2 \mathrm{~h}$ of OFF and were incubated for an additional 18-24 h at which time protein was harvested. Western blot analysis was performed to analyze OPN expression. Untransfected, lipofectamine only, and scramble siRNA treated osteoblasts all exhibited $\sim 2$.2-fold increase in OPN expression, whereas the FAK siRNAtreated osteoblasts only showed a 1.2-fold increase (Figs. $6 \mathrm{~A}$ and $6 \mathrm{~B})$. The expression of OPN in FAK siRNAtreated osteoblasts exposed to OFF was significantly decreased by $35-50 \%$ compared with the untransfected and control-treated osteoblasts exposed to OFF (Figs. 6A and $6 \mathrm{~B})$.

Overexpression of FRNK also disrupted FSS-stimulated upregulation of OPN. Uninfected and GFP only-infected osteoblasts exhibited a 2.0- to 2.2-fold increase in OPN expression after $2 \mathrm{~h}$ of OFF and an 18- to 24-h incubation (Figs. 6A and 6C). The FRNK-infected osteoblasts exhibited a 1.3-fold increase, which was not a significant increase in expression compared with the static control (Figs. $6 \mathrm{~A}$ and $6 \mathrm{C}$ ). This translated into a statistically significant $\sim 80 \%$ loss in OPN upregulation in the FRNK overexpressing osteoblasts.

Similarly, the $\mathrm{FAK}^{-/-}$clones exhibited a insignificant increase in OPN expression compared with $\mathrm{FAK}^{-1-}$ cells maintained in static culture conditions (Figs. 6A and 6D). The control clones exhibited a 2.7- to 4.7-fold increase in OPN expression compared with their static controls (Figs. $6 \mathrm{~A}$ and $6 \mathrm{D}$ ). This translated into a significant decrease of $\sim 50-70 \%$ in OPN upregulation. These findings indicate that FAK is needed for appropriate upregulation of OPN by FSS in osteoblasts.

We also analyzed protein samples for changes in FAK and Pyk2 expression after $2 \mathrm{~h}$ of OFF followed by an 18- to 24-h incubation in both the primary rat osteoblasts and the immortalized mouse osteoblast clones. We did not observe a significant change in FAK or Pyk2 expression as determined by Western blot analysis (data not shown). Furthermore, we did not observe a significant change in FAK or Pyk2 activation (Figs. 6E-6G).

\section{Re-expression of FAK in FAK $\mathrm{K}^{-1-}$ osteoblasts rescues $O F F$-induced $C O X-2$ and $O P N$ induction}

To re-express FAK in $\mathrm{FAK}^{-1-}$ osteoblast clone 4F1, we infected cells with FAK-GFP (4F1 + FAK). We also infected $\mathrm{FAK}^{-1-}$ osteoblasts with GFP only as a negative control (4F1 + GFP). Western blot analysis determined that the $4 \mathrm{~F} 1+$ FAK osteoblasts expressed FAK at $\sim 67 \%$ of wildtype compared with control osteoblasts, which was not a significant difference (Fig. 7A). We also determined that the $4 \mathrm{~F} 1+$ FAK osteoblasts exhibited $\sim 86 \%$ activation (measured as Y397 phosphorylation) compared with wildtype controls osteoblasts (Fig. 7A). Furthermore, reexpression of FAK in $\mathrm{FAK}^{-1-}$ osteoblasts did not change Pyk2 expression or activation (Fig. 7A).

We also analyzed protein samples for changes in FAK and Pyk2 expression after exposure to OFF. We did not observe a significant change in FAK or Pyk2 expression as 


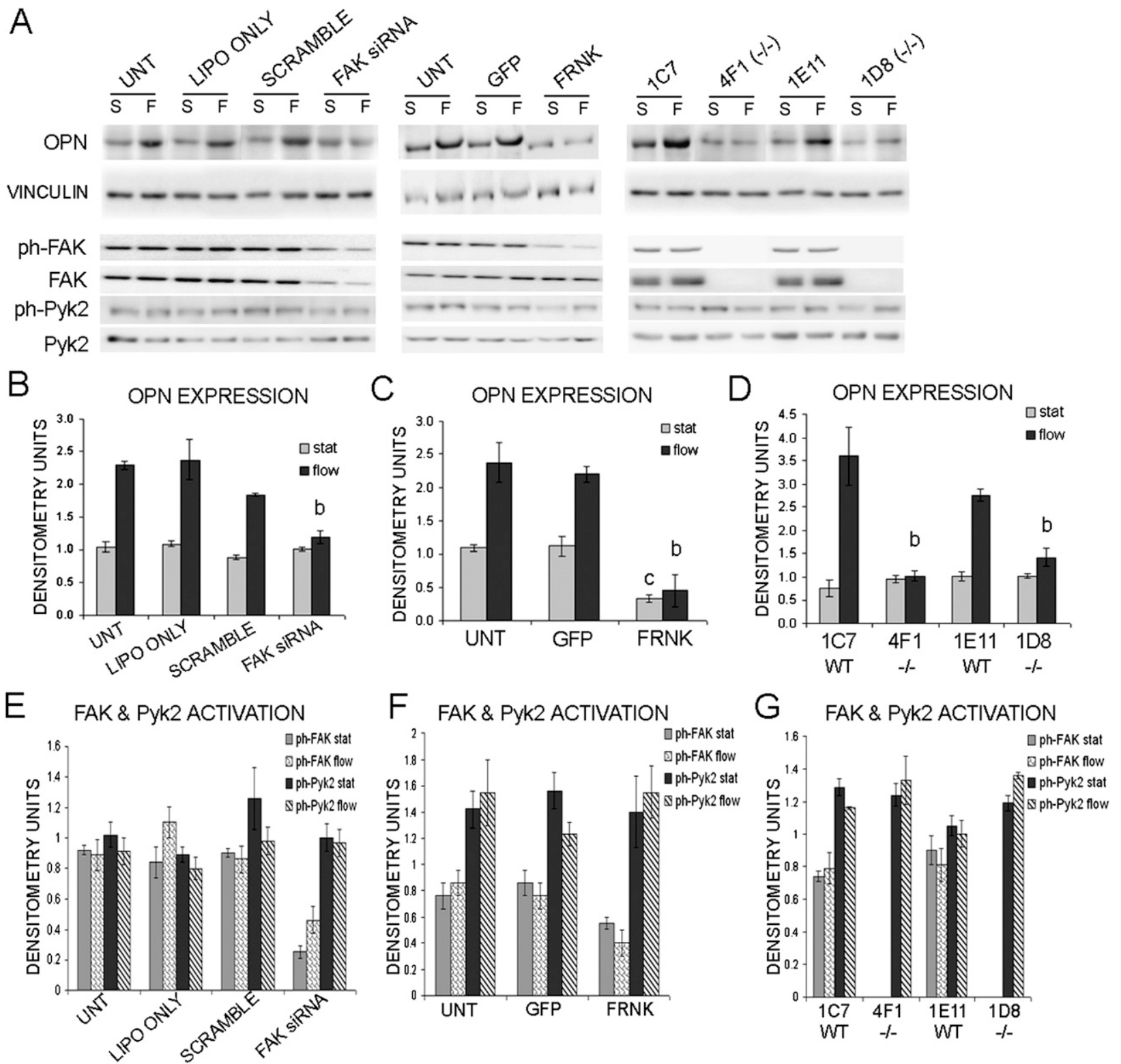

FIG. 6. Decreased OPN upregulation on exposure to OFF in osteoblasts with disrupted FAK activation. (A) Western blot analysis of OPN expression, FAK and Pyk 2 expression, and activation after $2 \mathrm{~h}$ of static culture conditions or $2 \mathrm{~h}$ of OFF both followed by an over night incubation (S, static; F, flow). Vinculin was analyzed as a loading control. (B-D) Quantification of OPN expression expressed in raw

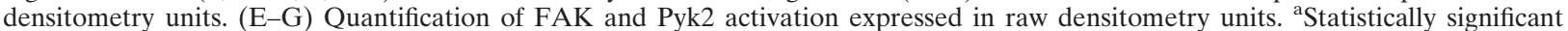
difference between static and flow. ${ }^{\mathrm{b}}$ Statistically significant difference among the flowed samples. ${ }^{\mathrm{c}}$ Statistically significant difference among the static samples $\left({ }^{a, b, c} p<0.05\right)$. Error bars represent SE.

determined by Western blot analysis (data not shown). Furthermore, we did not observe a significant change in FAK or Pyk2 activation (data not shown).

We next examined the 4F1 + FAK osteoblasts for the ability to upregulate COX-2 in response to OFF. Fortyeight hours after infection, cells were either maintained in static culture conditions or exposed to $2 \mathrm{~h}$ of OFF, and Western blot analysis was used to measure COX-2 expression. Re-expression of FAK in the $\mathrm{FAK}^{-1-}$ osteoblasts was able to rescue OFF-induced COX-2. Rescued osteoblasts exhibited a 2.9-fold increase in COX-2 expression in the $4 \mathrm{~F} 1+\mathrm{FAK}$ osteoblasts compared with a 1.3-fold induction in the 4F1 + GFP osteoblasts (Fig. 7B). The level of expression of COX-2 after exposure to OFF in the $4 \mathrm{~F} 1+$ FAK osteoblasts was not significantly different compared with wildtype controls osteoblasts (Fig. 7B).

In addition, we examined OPN protein expression in the $4 \mathrm{~F} 1+\mathrm{FAK}$ osteoblasts in response to $2 \mathrm{~h}$ of OFF followed by an 18 - to $24-\mathrm{h}$ incubation. Whereas the $4 \mathrm{~F} 1+\mathrm{GFP}$ control osteoblasts did not show a significant increase in OPN expression compared with static conditions, the 4F1+ FAK osteoblasts exhibited a significant $\sim 2$-fold increase in 


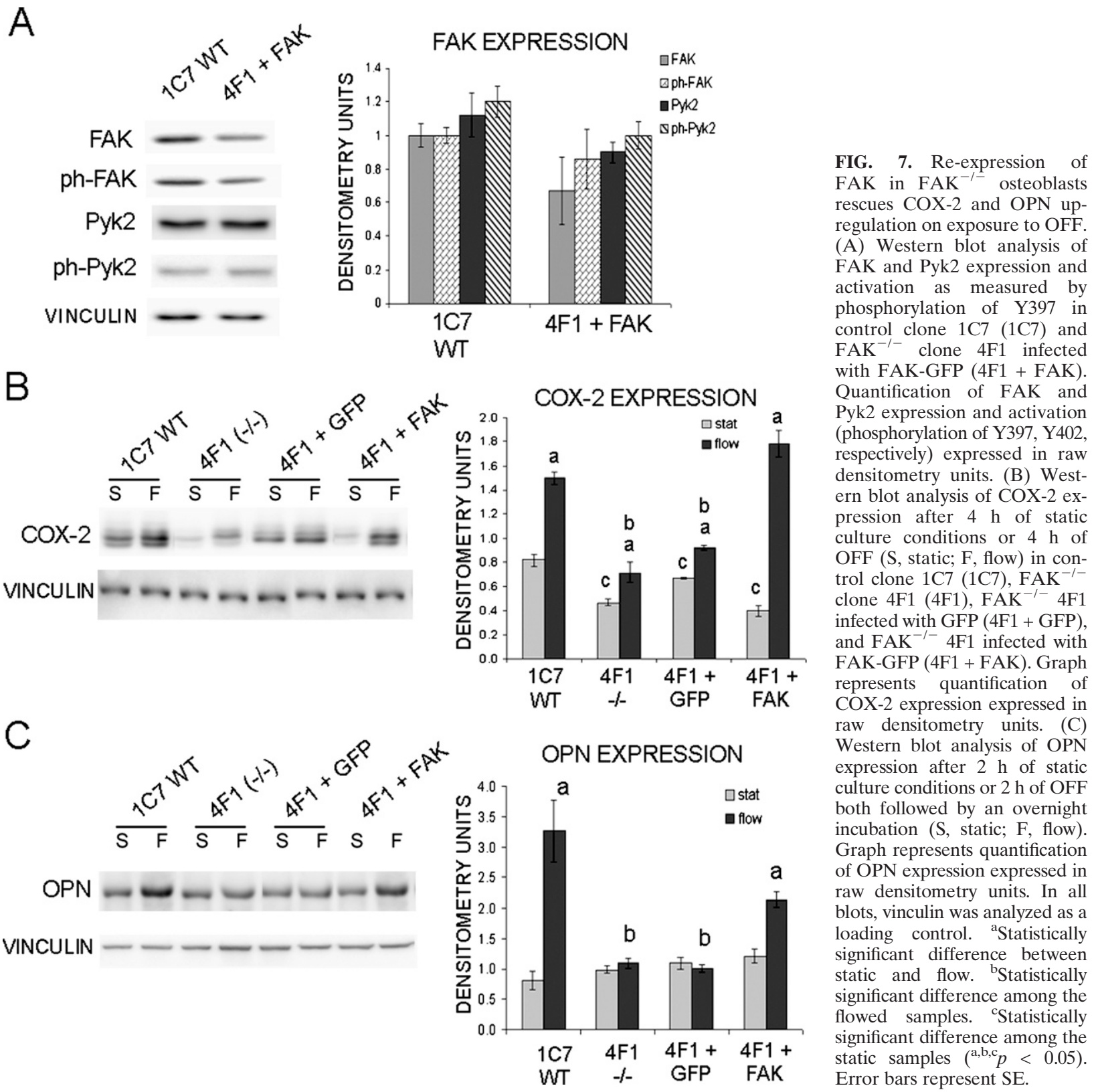

OPN expression after exposure to FSS (Fig. 7C). The wildtype controls also exhibited a significant increase in OPN expression after exposure to OFF, which was not significantly different to the level of OPN expression shown by the 4F1 + FAK osteoblasts after exposure to OFF (Fig. 7C).

\section{DISCUSSION}

Focal adhesions have been implicated as likely mechanosensors in bone cells. ${ }^{(5-9)}$ Our laboratory and others have shown the importance of focal adhesions in sensing and responding to FSS leading to upregulation of COX-2 and release of $\mathrm{PGE}_{2}$ from osteoblasts. ${ }^{(7,8,33,35,48-50)}$ In this study, we evaluate the molecular mechanisms through which extracellular mechanical signals are transduced across focal adhesions. We examined the role of a single component of the focal adhesion, FAK, to better understand how focal adhesions mediate mechanotransduction in osteoblasts. We showed that FAK activation, as measured by phosphorylation of Y397, can be disrupted by decreasing FAK expression using siRNA and by overexpression of the dominant negative FAK protein FRNK. In addition, we were able to analyze $\mathrm{FAK}^{-1-}$ osteoblast clones established from conditional $\mathrm{fak}^{-/-}$mice. ${ }^{(21)}$ Each of our three methods of disrupting FAK activation were capable of impairing mechanotransduction as measured by several endpoints after periods of OFF, indicating a correlation between the level of activated FAK and impaired 
OFF-induced mechanotransduction. Importantly, the critical role of FAK in OFF-induced osteoblast mechanotransduction was evident over a broad range of time courses ranging from 5 to $15 \mathrm{~min}$ in the case of ERK activation to 24 $\mathrm{h}$ in the case of OPN expression. These results indicate that FAK plays a critical role in shear-induced mechanotransduction in osteoblasts.

To study the role of FAK in an early response to OFF, we examined ERK phosphorylation after 5, 15, and $30 \mathrm{~min}$ of OFF in osteoblasts. We found that each of the three methods used to disrupt FAK function resulted in reduced ERK phosphorylation at 5 and 15 min of OFF. Many laboratories have shown that shear stress activates ERK, and ERK phosphorylation is important for the regulation of cell proliferation and is also involved in gene regulation, differentiation, and regulation of apoptosis..$^{(8,29-34,38,51,52)}$ This report showed that FAK activation through OFF is upstream of ERK phosphorylation in osteoblasts. These results are similar to results obtained by Li et al., ${ }^{(53)}$ which showed FAK activation upstream of ERK phosphorylation in endothelial cells is mediated by the Grb2-Sos pathway. ${ }^{(53)}$ In addition, several laboratories have shown the disruption of ERK activation on expression of FRNK. ${ }^{(54-57)}$

We also examined the role of FAK during OFF regulation of gene expression in osteoblasts. Using each of our three methods to disrupt FAK activation, FAK was found to be important for the induction of c-fos, COX-2, and OPN expression by OFF in osteoblasts. Because the time course required for optimal induction of c-fos, COX-2, and OPN differs significantly, ranging from 1 to $24 \mathrm{~h}$, analysis of expression of these three proteins suggests a role of FAK in mechanotransduction that is not temporally limited to a brief or specific period of OFF. In addition, we found that FAK activity is also important for the OFF induction of $\mathrm{PGE}_{2}$. Whereas other studies have shown that expression of c-fos, ${ }^{(36-38,41,58)} \mathrm{COX}-2,{ }^{(7,8,33,35,37,48,59)}$ and $\mathrm{OPN}^{(34,47)}$ is regulated by shear stress, this study is the first to show a relationship between FAK activity and the regulation of expression of these proteins.

$\mathrm{FAK}^{-1-}$ osteoblasts did exhibit a small but significant increase in c-fos and COX-2 expression and ERK activation after exposure to OFF, suggesting that these cells were able to detect and respond to FSS, albeit at a lesser degree than their control counterparts. We showed that, in osteoblasts, the main source of signaling induced by OFF occurs through the focal adhesion complex and FAK. However, we speculate that other molecules also respond to FSS in parallel to FAK, resulting in the reduced, but not absent, response to OFF observed in $\mathrm{FAK}^{-1-}$ osteoblasts. Lee et al. ${ }^{(60)}$ showed integrin-dependent FSS activation of Shc in osteoblasts, which is linked to activation of ERK and regulation of c-fos, COX-2, and OPN expression. Perhaps this pathway works in parallel with FSS-induced FAK signaling to regulate anabolic osteoblast activity. Additionally, there is evidence that integrins can activate MAPK independent of FAK activity, ${ }^{(61)}$ indicating another mechanism that may contribute to the $\mathrm{FAK}^{-1-}$ osteoblasts to response to OFF. Calcium signaling may also be playing a role in FSS-induced mechanotransduction in the $\mathrm{FAK}^{-1-}$ osteoblasts. ${ }^{(62)}$ FSS stimulates increased intracellular cal- cium in osteoblasts, which can mediate $\mathrm{PGE}_{2}$ release and COX-2 and c-fos expression. ${ }^{(37,44)}$

FAK is a member of the focal adhesion protein nonreceptor tyrosine kinase family along with Pyk2. ${ }^{(63)}$ Several reports have shown that FAK and Pyk2 have common phosphorylation targets such as ERK and Shc, and both are implicated in signaling through $\mathrm{p} 130^{\text {cas }}$ (63) $^{6}$ Whereas the deletion of fak in mice resulted in an embryonic lethal phenotype, $P y k 2^{-1-}$ mice exhibited an increased bone mass, increased bone formation activity, and defects in osteoclast function. ${ }^{(64-66)}$ Because of the data implicating the importance of Pyk2 in anabolic osteoblast activity, we examined the expression and activation of Pyk2 in the siRNA-treated osteoblasts, viral-transduced osteoblasts, and $\mathrm{FAK}^{-1-}$ osteoblasts clones compared with the control clones. We did not find a significant change in Pyk2 expression or Pyk 2 activation (measure by phosphorylation of tyrosine 402) resulting from any of the methods we used to target FAK expression and activation. Whereas it has recently been reported that these $\mathrm{FAK}^{-1-}$ clones do exhibit an increase in Pyk2 activation, our laboratory could not detect such a change. ${ }^{(21)}$ The variability among the $\mathrm{FAK}^{-1-}$ clones that have been analyzed by our laboratory and others could possibly explain the difference in Pyk2 expression and activation. Also, the method in which protein samples were harvested varied between laboratories and therefore could contribute to differences in Pyk2 protein levels detected between samples. Whereas we did not detect a difference in Pyk2 expression or activation, this does not rule out the possibility that Pyk2 activity is involved in the mechanotransduction pathways in osteoblasts.

Additionally, we examined the effect of OFF on FAK and Pyk 2 expression and activation. We determined that OFF did not change FAK or Pyk2 expression in osteoblasts. Furthermore, we showed that OFF did not change FAK and Pyk2 activation as measured by phosphorylation of Y397 and Y402, respectively. Whereas we did not detect a change in activation of FAK or Pyk2 with short and long periods of OFF, we do not disregard the possibility that there is an immediate increase in FAK and/or Pyk2 activation after exposure to OFF that we cannot detect because of the speed at which it occurs. We speculate that basal levels of activated FAK are sufficient to maintain appropriate OFF-induced mechanotransduction in osteoblasts, but we maintain that possible immediate increases maybe occurring.

Previous work in our laboratory showed that blocking the linkage of stress fibers to integrins at focal adhesions by microinjection of a dominant fragment of $\alpha$-actinin or by inhibition of Rho activity inhibited FSS induction of COX2 and c-fos expression in osteoblasts. ${ }^{(7)} \mathrm{We}$ also found that pharmacologic disruption of actin filaments, microtubules, or intermediate filaments was not sufficient to inhibit FSS induced $\mathrm{PGE}_{2}$ release or COX-2 expression in osteoblasts. ${ }^{(8)}$ Whereas this study concluded that disruption of any one of these cytoskeletal networks did not inhibit FSS mechanotransduction, it does not imply that a functional cytoskeletal network might not be an important component in other aspects of mechanotransduction. Our data 
reported in this study implicate the focal adhesion as a critical component of mechanotransduction in osteoblasts.

Previously it was reported that FAK is important for bone healing and remodeling in mice. ${ }^{(21)}$ It was determined that $\mathrm{FAK}^{-1-}$ osteoblasts were able to differentiate but exhibited a bone regeneration defect including slow deposition of extracellular matrix and large callus formation. ${ }^{(21)}$ Our data suggest that these $\mathrm{FAK}^{-/-}$osteoblasts cells cannot properly respond to the mechanical signals produced during bone regeneration and remodeling resulting in delayed and incomplete repair of the damaged site. Further support for this hypothesis is shown by in vivo data from the Helms laboratory, which also used the conditional $\mathrm{fak}^{-/-}$mouse model and showed that bone marrow cells need FAK function for appropriate response to mechanical stimuli generated by a micromotion device. ${ }^{(67)}$ Furthermore, because FAK has many binding partners, it is a good candidate for mediating the mechanical signal of OFF, which stimulates a variety of anabolic effects. We speculate that multiple effectors of FAK will be involved in any one endpoint examined, and further studies will need to be performed to delineate which pathways are involved with each different endpoint. In addition, we hypothesize that other integrin binding proteins may also be involved in OFF-induced mechanotransduction but to a lesser degree than FAK. Our data, in concert with data from the Helms laboratory, suggest that FAK is a key regulator for mechanical signaling induced by FSS in osteoblasts. Further work will be needed to understand the downstream effectors of FAK transduce the external mechanical stimulus of OFF into anabolic changes in the cell.

In summary, we examined the role of FAK during OFFinduced mechanotransduction in osteoblasts. Using three methods of disrupting FAK function, we determined that FAK is important for the OFF-induced activation of ERK and the OFF-induced expression of c-fos, COX-2, OPN, and $\mathrm{PGE}_{2}$ release. This study is the first to begin exploring the molecular mechanisms at focal adhesions that are involved in OFF-induced mechanotransduction in osteoblasts.

\section{ACKNOWLEDGMENTS}

The authors thank Suzanne Ponik and Eric Rodenberg for helpful discussions and suggestions. This study was funded by NIH Grants AR049728 and AR052682.

\section{REFERENCES}

1. Harder AT, An YH, 2003 The mechanisms of the inhibitory effects of nonsteroidal anti-inflammatory drugs on bone healing: A concise review. J Clin Pharmacol 43:807-815.

2. Miller SB 2006 Prostaglandins in health and disease: An overview. Semin Arthritis Rheum 36:37-49.

3. Radi ZA, Khan NK, 2005 Effects of cyclooxygenase inhibition on bone, tendon, and ligament healing. Inflamm Res 54:358366.

4. Raisz LG 2001 Potential impact of selective cyclooxygenase-2 inhibitors on bone metabolism in health and disease. Am J Med 110(Suppl 3A):43S-45S.

5. Guignandon A, Akhouayri O, Usson Y, Rattner A, Laroche N, Lafage-Proust MH, Alexandre C, Vico L 2003 Focal contact clustering in osteoblastic cells under mechanical stresses:
Microgravity and cyclic deformation. Cell Commun Adhes 10:69-83.

6. Guignandon A, Boutahar N, Rattner A, Vico L, LafageProust MH 2006 Cyclic strain promotes shuttling of PYK2/ Hic-5 complex from focal contacts in osteoblast-like cells. Biochem Biophys Res Commun 343:407-414.

7. Pavalko FM, Chen NX, Turner CH, Burr DB, Atkinson S, Hsieh YF, Qiu J, Duncan RL 1998 Fluid shear-induced mechanical signaling in MC3T3-E1 osteoblasts requires cytoskeleton-integrin interactions. Am J Physiol 275:C1591-C1601.

8. Norvell SM, Ponik SM, Bowen DK, Gerard R, Pavalko FM 2004 Fluid shear stress induction of COX-2 protein and prostaglandin release in cultured MC3T3-E1 osteoblasts does not require intact microfilaments or microtubules. J Appl Physiol 96:957-966.

9. Wozniak M, Fausto A, Carron CP, Meyer DM, Hruska KA 2000 Mechanically strained cells of the osteoblast lineage organize their extracellular matrix through unique sites of alphavbeta3-integrin expression. J Bone Miner Res 15:17311745 .

10. Boudreau NJ, Jones PL, 1999 Extracellular matrix and integrin signalling: The shape of things to come. Biochem $\mathrm{J}$ 339:481-488.

11. Critchley DR 2000 Focal adhesions: The cytoskeletal connection. Curr Opin Cell Biol 12:133-139.

12. Damsky CH, Ilic D, 2002 Integrin signaling: It's where the action is. Curr Opin Cell Biol 14:594-602.

13. Miranti CK, Brugge JS, 2002 Sensing the environment: A historical perspective on integrin signal transduction. Nat Cell Biol 4:E83-E90.

14. Calalb MB, Polte TR, Hanks SK 1995 Tyrosine phosphorylation of focal adhesion kinase at sites in the catalytic domain regulates kinase activity: A role for Src family kinases. Mol Cell Biol 15:954-963.

15. Schaller M, Hildebrand J, Shannon J, Fox J, Vines R, Parsons J 1994 Autophosphorylation of the focal adhesion kinase, pp125FAK, directs SH2-dependent binding of pp60src. Mol Cell Biol 14:1680-1688.

16. Xing Z, Chen H, Nowlen J, Taylor S, Shalloway D, Guan J 1994 Direct interaction of v-Src with the focal adhesion kinase mediated by Src SH2 domain. Mol Cell Biol 5:413-421.

17. Guan JL 1997 Focal adhesion kinase in integrin signaling. Matrix Biol 16:195-200.

18. Parsons JT 2003 Focal adhesion kinase: The first ten years. J Cell Sci 116:1409-1416.

19. Mitra SK, Schlaepfer DD, 2006 Integrin-regulated FAK-Src signaling in normal and cancer cells. Curr Opin Cell Biol 18:516-523.

20. Playford MP, Schaller MD, 2004 The interplay between Src and integrins in normal and tumor biology. Oncogene 23: 7928-7946.

21. Kim JB, Leucht P, Luppen CA, Park YJ, Beggs HE, Damsky $\mathrm{CH}$, Helms JA 2007 Reconciling the roles of FAK in osteoblast differentiation, osteoclast remodeling, and bone regeneration. Bone 41:39-51.

22. Jackson M, Shalhoub V, Lian J, Stein G, Marks S Jr 1994 Aberrant gene expression in cultured mammalian bone cells demonstrates an osteoblast defect in osteopetrosis. J Cell Biochem 55:366-372.

23. Triplett JW, Pavalko FM 2006 Disruption of alpha-actininintegrin interactions at focal adhesions renders osteoblasts susceptible to apoptosis. Am J Physiol Cell Physiol 291:C909C921.

24. Jacobs CR, Yellowley CE, Davis BR, Zhou Z, Cimbala JM, Donahue HJ 1998 Differential effect of steady versus oscillating flow on bone cells. J Biomech 31:969-976.

25. Sheffield JB, Graff D, Li HP 1987 A solid-phase method for the quantitation of protein in the presence of sodium dodecyl sulfate and other interfering substances. Anal Biochem 166: $49-54$.

26. Richardson A, Parsons T 1996 A mechanism for regulation of the adhesion-associated proteintyrosine kinase pp125FAK. Nature 380:538-540. 
27. Schaller MD, Borgman CA, Parsons JT 1993 Autonomous expression of a noncatalytic domain of the focal adhesionassociated protein tyrosine kinase pp125FAK. Mol Cell Biol 13:785-791.

28. Schlaepfer DD, Hunter T 1998 Integrin signalling and tyrosine phosphorylation: Just the FAKs? Trends Cell Biol 8: 151-157.

29. Jackson RA, Kumarasuriyar A, Nurcombe V, Cool SM 2006 Long-term loading inhibits ERK1/2 phosphorylation and increases FGFR3 expression in MC3T3-E1 osteoblast cells. J Cell Physiol 209:894-904.

30. Jiang GL, White CR, Stevens HY, Frangos JA 2002 Temporal gradients in shear stimulate osteoblastic proliferation via ERK1/2 and retinoblastoma protein. Am J Physiol Endocrinol Metab 283:E383-E389.

31. Kapur S, Baylink DJ, Lau KH 2003 Fluid flow shear stress stimulates human osteoblast proliferation and differentiation through multiple interacting and competing signal transduction pathways. Bone 32:241-251.

32. Kapur S, Chen ST, Baylink DJ, Lau KH 2004 Extracellular signal-regulated kinase- 1 and -2 are both essential for the shear stress-induced human osteoblast proliferation. Bone 35: $525-534$.

33. Wadhwa S, Godwin SL, Peterson DR, Epstein MA, Raisz LG, Pilbeam CC 2002 Fluid flow induction of cyclo-oxygenase 2 gene expression in osteoblasts is dependent on an extracellular signal-regulated kinase signaling pathway. J Bone Miner Res 17:266-274

34. You J, Reilly GC, Zhen X, Yellowley CE, Chen Q, Donahue HJ, Jacobs CR 2001 Osteopontin gene regulation by oscillatory fluid flow via intracellular calcium mobilization and activation of mitogen-activated protein kinase in MC3T3-E1 osteoblasts. J Biol Chem 276:13365-13371.

35. Ponik SM, Triplett JW, Pavalko FM 2007 Osteoblasts and osteocytes respond differently to oscillatory and unidirectional fluid flow profiles. J Cell Biochem 100:794-807.

36. Charoonpatrapong-Panyayong K, Shah R, Yang J, Alvarez M, Pavalko FM, Gerard-O'riley R, Robling AG, Templeton E, Bidwell JP 2007 Nmp4/CIZ contributes to fluid shear stress induced MMP-13 gene induction in osteoblasts. J Cell Biochem 4:13-25.

37. Chen NX, Ryder KD, Pavalko FM, Turner CH, Burr DB, Qiu J, Duncan RL $2000 \mathrm{Ca}(2+)$ regulates fluid shear-induced cytoskeletal reorganization and gene expression in osteoblasts. Am J Physiol Cell Physiol 278:C989-C997.

38. Ferraro JT, Daneshmand M, Bizios R, Rizzo V 2004 Depletion of plasma membrane cholesterol dampens hydrostatic pressure and shear stress-induced mechanotransduction pathways in osteoblast cultures. Am J Physiol Cell Physiol 286:C831C839.

39. Nomura S, Takano-Yamamoto T 2000 Molecular events caused by mechanical stress in bone. Matrix Biol 19:91-96.

40. Peake MA, El Haj AJ 2003 Preliminary characterisation of mechanoresponsive regions of the c-fos promoter in bone cells. FEBS Lett 537:117-120.

41. Tanaka SM, Sun HB, Roeder RK, Burr DB, Turner CH, Yokota H 2005 Osteoblast responses one hour after loadinduced fluid flow in a three-dimensional porous matrix. Calcif Tissue Int 76:261-271.

42. Bakker AD, Soejima K, Klein-Nulend J, Burger EH 2001 The production of nitric oxide and prostaglandin $\mathrm{E}(2)$ by primary bone cells is shear stress dependent. J Biomech $\mathbf{3 4}$ : 671-677.

43. Klein-Nulend J, Burger EH, Semeins CM, Raisz LG, Pilbeam CC 1997 Pulsating fluid flow stimulates prostaglandin release and inducible prostaglandin $\mathrm{G} / \mathrm{H}$ synthase mRNA expression in primary mouse bone cells. J Bone Miner Res 12:45-51.

44. Reich KM, Frangos JA 1991 Effect of flow on prostaglandin E2 and inositol trisphosphate levels in osteoblasts. Am J Physiol 261:C428-C432.

45. Reich KM, McAllister TN, Gudi S, Frangos JA 1997 Activation of $\mathrm{G}$ proteins mediates flow-induced prostaglandin E2 production in osteoblasts. Endocrinology 138:1014-1018.
46. Smalt R, Mitchell FT, Howard RL, Chambers TJ 1997 Mechanotransduction in bone cells: Induction of nitric oxide and prostaglandin synthesis by fluid shear stress, but not by mechanical strain. Adv Exp Med Biol 433:311-314.

47. Batra NN, Li YJ, Yellowley CE, You L, Malone AMD, Kim $\mathrm{CH}$, Jacobs CR 2005 Effects of short-term recovery periods on fluid-induced signaling in osteoblastic cells. J Biomech 38: 1909-1917.

48. Ogasawara A, Arakawa T, Kaneda T, Takuma T, Sato T, Kaneko H, Kumegawa M, Hakeda Y 2001 Fluid shear stressinduced cyclooxygenase-2 expression is mediated by C/EBP beta, cAMP-response element-binding protein, and AP-1 in osteoblastic MC3T3-E1 cells. J Biol Chem 276:7048-7054.

49. Ponik SM, Pavalko FM 2004 Formation of focal adhesions on fibronectin promotes fluid shear stress induction of COX-2 and PGE2 release in MC3T3-E1 osteoblasts. J Appl Physiol 97: 135-142.

50. Wadhwa S, Choudhary S, Voznesensky M, Epstein M, Raisz L, Pilbeam C 2002 Fluid flow induces COX-2 expression in MC3T3-E1 osteoblasts via a PKA signaling pathway. Biochem Biophys Res Commun 297:46-51.

51. Mehrotra M, Saegusa M, Wadhwa S, Voznesensky O, Peterson D, Pilbeam C 2006 Fluid flow induces Rankl expression in primary murine calvarial osteoblasts. J Cell Biochem 98:1271-1283.

52. Triplett JW, O'Riley R, Tekulve K, Norvell SM, Pavalko FM 2007 Mechanical loading by fluid shear stress enhances IGF1 receptor signaling in osteoblasts in a PKCzeta-dependent manner. Mol Cell Biomech 4:13-25.

53. Li S, Kim M, Hu T, Jalali S, Schlaepfer D, Hunter T, Chien S, Shyy J 1997 Fluid shear stress activation of focal adhesion kinase. Linking to mitogen-activated protein kinases. J Biol Chem 48:30455-30462.

54. Hauck CR, Hsia DA, Puente XS, Cheresh DA, Schlaepfer DD 2002 FRNK blocks v-Src-stimulated invasion and experimental metastases without effects on cell motility or growth. EMBO J 21:6289-6302.

55. Lu KK, Armstrong SE, Ginnan R, Singer HA 2005 Adhesiondependent activation of CaMKII and regulation of ERK activation in vascular smooth muscle. Am J Physiol Cell Physiol 289:C1343-C1350.

56. Neff L, Zeisel M, Druet V, Takeda K, Klein JP, Sibilia J, Wachsmann D 2003 ERK 1/2- and JNKs-dependent synthesis of interleukins 6 and 8 by fibroblast-like synoviocytes stimulated with protein I/II, a modulin from oral streptococci, requires focal adhesion kinase. J Biol Chem 278:27721-27728.

57. Sawhney RS, Cookson MM, Omar Y, Hauser J, Brattain MG 2006 Integrin alpha2-mediated ERK and calpain activation play a critical role in cell adhesion and motility via focal adhesion kinase signaling: Identification of a novel signaling pathway. J Biol Chem 281:8497-8510.

58. Inoue D, Kido S, Matsumoto T 2004 Transcriptional induction of FosB/DeltaFosB gene by mechanical stress in osteoblasts. J Biol Chem 279:49795-49803.

59. Mehrotra M, Saegusa M, Voznesensky O, Pilbeam C 2006 Role of Cbfa1/Runx2 in the fluid shear stress induction of COX-2 in osteoblasts. Biochem Biophys Res Commun 341:1225-1230.

60. Lee DY, Yeh CR, Chang SF, Lee PL, Chien S, Cheng CK, Chiu JJ 2008 Integrin-mediated expression of bone formationrelated genes in osteoblast-like cells in response to fluid shear stress: Roles of extracellular matrix, Shc, and mitogen-activated protein kinase. J Bone Miner Res 23:1140-1149.

61. Juliano R 1996 Cooperation between soluble factors and integrin-mediated cell anchorage in the control of cell growth and differentiation. Bioessays 18:911-917.

62. Hung CT, Allen FD, Pollack SR, Brighton CT 1996 Intracellular $\mathrm{Ca} 2+$ stores and extracellular $\mathrm{Ca} 2+$ are required in the real-time $\mathrm{Ca} 2+$ response of bone cells experiencing fluid flow. J Biomech 29:1411-1417.

63. Avraham H, Park SY, Schinkmann K, Avraham S 2000 RAFTK/Pyk2-mediated cellular signalling. Cell Signal 12: 123-133. 
64. Buckbinder L, Crawford DT, Qi H, Ke HZ, Olson LM, Long KR, Bonnette PC, Baumann AP, Hambor JE, Grasser WA III, Pan LC, Owen TA, Luzzio MJ, Hulford CA, Gebhard DF, Paralkar VM, Simmons HA, Kath JC, Roberts WG, Smock SL, Guzman-Perez A, Brown TA, Li M 2007 Proline-rich tyrosine kinase 2 regulates osteoprogenitor cells and bone formation, and offers an anabolic treatment approach for osteoporosis. Proc Natl Acad Sci USA 104:10619-10624.

65. Gil-Henn H, Destaing O, Sims NA, Aoki K, Alles N, Neff L, Sanjay A, Bruzzaniti A, De Camilli P, Baron R, Schlessinger J 2007 Defective microtubule-dependent podosome organization in osteoclasts leads to increased bone density in Pyk2(-/-) mice. J Cell Biol 178:1053-1064.

66. Ilic D, Furuta Y, Suda T, Atsumi T, Fujimoto J, Ikawa Y, Yamamoto T, Aizawa S 1995 Focal adhesion kinase is not essential for in vitro and in vivo differentiation of ES cells. Biochem Biophys Res Commun 209:300-309.
67. Leucht P, Kim JB, Currey JA, Brunski J, Helms JA 2007 FAKMediated Mechanotransduction in Skeletal Regeneration. PLoS One 2:e390.

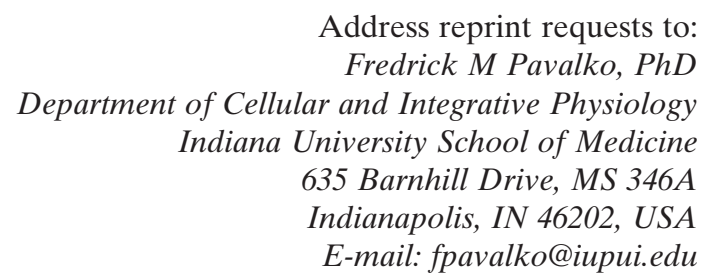

Received in original form May 23, 2008; revised form September 23, 2008; accepted October 28, 2008. 